\title{
Multiple Attribute Grey Target Decision Making Model Based on Linear Combination Weights Determining and Entropy
}

\author{
Zhihong Gu \& Dongxiao Niu \\ School Of Business Administration, North China Electric Power University, Beijing 102206, China \\ Tel: 86-10-5196-3675 E-mail: laobing618@126.com \\ Huiqing Wang \\ School of Economy and Business Administration, Shanxi University, Taiyuan 030006, China \\ E-mail: wanghuiqing98213@163.com
}

\begin{abstract}
Gray target decision making is one method of gray systematic theory for solving multiple attribute decision making problem. The weight definite method of the index in the present researches on gray target decision making is monotonous, it does not accord with the actual conditions; From the point of view of mathematical statistics in addition, the true weight coefficient of every index is a random variable, the weight coefficient that different assignment method draws is only a sample value of the true weight coefficient, and it has some uncertainty. For this reason, a method of determining the linear combination weights based on entropy, using optimization theory and Jayne's maximum entropy principle, is presented to deal with the problem of determining the weights in multiple attribute decision making. The existing grey target decision making model has been got improved and the example demonstrates the validity of this model.
\end{abstract}

Keywords: Entropy, Grey target decision making, Linear combination assigning weights, Multiple attribute decision making

\section{Forewords}

There are a large number of MADM (Multiple Attribute Decision Making) problems in such activities as the society, economy and etc. A lot of domestic and international scholars have already carried on diversified researches and discussions to MADM and have made it develop rapidly. The grey target decision making is one of the methods to solve MADM problems using the grey systematic theory. An important factor influencing the rationality of MADM is the weight of evaluation index. The existing researches of grey target decision making (documents [1, 2]) all treat the importance of every index equally, or assign weight to every index with single subjective law. In reality, different indexes have different function in different decision, so it dose not accord with actual conditions to treat the importance of every index equally or assign weight with single subjective law and it is essential for improvement of the already existing grey target decision making model. From the point of view of mathematical statistics in addition, the true weighting coefficient of every index is a random variable, the weighting coefficient that different assignment method draws is only a sample value of the true weighting coefficient, and it has some uncertainty. For this reason, this text proposes one method giving consideration to the synthesis of subjective law and objective law, introduces Shannon entropy to describe the uncertainty brought by various kinds of assignment methods at the same time, and on this basis has set up the multiple attribute grey target decision making model. The instance analysis with this model has stated the rationality of it.

\section{Set up of the grey target decision making model}

Generally a MADM problem has a decision scheme set $S=\left\{s_{1}, s_{2}, \mathrm{~L}, s_{n}\right\}$, which is composed by assessed targets or drafted schemes; and an index set $A=\left\{a_{1}, a_{2}, \mathrm{~L}, a_{m}\right\}$, which is composed by evaluation indexes or attributes. The effect sample value of the scheme $s_{i}$ to the index $a_{j}$ is $x_{i j}(i=1,2, \mathrm{~L}, n ; j=1,2, \mathrm{~L}, m)$ and the effect sample matrix of the scheme set $S$ to the index set $A$ is $X=\left[x_{i j}\right]_{n \times m}$.

\subsection{Index standardization}

Because the magnitude order and dimension of every evaluation index will exist differently in actual appraisal problem and the attribute is different, some indexes require that the smaller the better, some indexes require that the greater the better, while some indexes demand to fall into a certain interval. So it is necessary to carry on the standardized treatment to the appraise matrix before appraising.

At present the commonly used index types include benefit type, cost type and interval type, etc. It is the better that benefit type index value is the greater, the better that cost type index value is the smaller, and the better that interval 
type index value falls into a certain interval. This text will use the principle of "reward the good and punish the bad" to standardize indexes according to reference document (Dai, Zou, Wang, Zhu \& Zhou, 2000, pp. 32-36), the basic thought is that a positive number from 0 to 1 is assigned to the index when its value is superior to the average level, while a negative number from 0 to -1 is assigned to the index when its value is inferior to the average level.

It is supposed that $\mathrm{O}_{1}, \mathrm{O}_{2}, \mathrm{O}_{3}$ respectively stands for the suffix sets of benefit type index, cost type index and interval type index, so there is the equation $O_{1} \mathrm{U} O_{2} \mathrm{U} O_{3}=\{1,2, \mathrm{~L}, m\}$, and $O_{i} \mathrm{I} O_{j}=\varnothing(i \neq j, i, j=1,2,3)$.

Order $z_{j}=\frac{1}{n} \sum_{i=1}^{n} x_{i j},(j=1,2, \mathrm{~L}, m)$, if $A_{j}$ is benefit type index, there is the formula (1):

$$
r_{i j}=\frac{x_{i j}-z_{j}}{\max \left(\max _{1 \leq i \leq n}\left\{x_{i j}\right\}-z_{j}, z_{j}-\min _{1 \leq i \leq n}\left\{x_{i j}\right\}\right)},(j=1,2, \mathrm{~L}, m)
$$

If $A_{j}$ is cost type index, there is the formula (2):

$$
r_{i j}=\frac{z_{j}-x_{i j}}{\max \left(\max _{1 \leq i \leq n}\left\{x_{i j}\right\}-z_{j}, z_{j}-\min _{1 \leq i \leq n}\left\{x_{i j}\right\}\right)},(j=1,2, \mathrm{~L}, m)
$$

And if $A_{j}$ is interval type index, for example, $A_{j}$ belongs to the interval of $[C, D]$, there is the formula (3):

$$
r_{i j}=\left\{\begin{array}{c}
1-\frac{2\left(C-x_{i j}\right)}{C-\min _{1 \leq i \leq n}\left\{x_{i j}\right\}}, x_{i j}<C \\
1-\frac{2\left(x_{i j}-D\right)}{\min _{1 \leq i \leq n}\left\{x_{i j}\right\}-D}, x_{i j}>C \\
1, \quad C \leq x_{i j} \leq D
\end{array} \quad(j=1,2, \mathrm{~L}, m)\right.
$$

The above transform is called the "reward the good and punish the bad" transform operator, and it can transform the effect sample matrix $X(t)=\left(x_{i j}\right)$ into the decision making matrix $R=\left[r_{i j}\right]_{n \times m}$. It can be concluded that all the elements of the matrix $R$ have no dimension and they all accord with the "reward the good and punish the bad" criterion, and that the value of every element belongs to the interval of [-1,1], $(i=1,2, \mathrm{~L}, n ; j=1,2, \mathrm{~L}, m)$. The vector $r_{i}=\left(r_{i 1}, r_{i 2}, \mathrm{~L}, r_{i m}\right)(i=1,2, \mathrm{~L}, n)$ is called the effect vector of the scheme $s_{i}$.

\subsection{The multiple attribute grey target decision making model}

(1) Definition 1: Supposed that

$$
r_{j}^{*}=\max _{1 \leq i \leq n}\left\{r_{i j}\right\},(j=1,2, \mathrm{~L}, m)
$$

Call the follow vector as the optimum effect vector for the multiple attribute grey target decision making, which is also called the "bull's-eye".

$$
r=\left(r_{1}^{*}, r_{2}^{*}, \mathrm{~L}, r_{m}^{*}\right)
$$

(2) Supposed that the vector of weight drawn through the method of linear combination weights assigning which is adopted by this text is $W^{*}=\left(w_{1}{ }^{*}, w_{2}{ }^{*}, \mathrm{~L}, w_{m}{ }^{*}\right)$.

Definition 2: Call the follow formula as the multidimensional ellipsoid grey target regarding $r$ as the "bull's-eye".

$$
R^{(m)}=\left\{\left(r_{i 1}, r_{i 2}, \mathrm{~L}, r_{i m}\right) \mid w_{1}^{*}\left(r_{i 1}-r_{1}^{*}\right)^{2}+w_{2}^{*}\left(r_{i 2}-r_{2}^{*}\right)^{2}+\mathrm{L}+w_{m}^{*}\left(r_{i m}-r_{m}^{*}\right)^{2}=R^{2}\right\}
$$

(3) Definition 3: Supposed that $r_{i}=\left(r_{i 1}, r_{i 2}, \mathrm{~L}, r_{i m}\right) \in R^{(m)}$, and call the variable $d_{i}$ as the "bull's-eye distance" of the 
effect vector $r_{i}$. The size of the "bull's-eye distance" has reflected the quality with the effect vector. The smaller the "bull's-eye distance" is, the more excellent the decision scheme is; On the contrary, the greater the "bull's-eye distance" is, the worse the decision scheme is.

$$
d_{i}=\left|r-r_{i}\right|=\sqrt{w_{1}^{*}\left(r_{i 1}-r_{1}^{*}\right)^{2}+w_{2}^{*}\left(r_{i 2}-r_{2}^{*}\right)^{2}+\mathrm{L} w_{m}^{*}\left(r_{i m}-r_{m}^{*}\right)^{2}}
$$

\subsection{The linear combination assigning weights based on entropy}

(1) Supposed that the appraising person gets several kinds of weight vectors for the evaluation index, such as $W^{1}, \ldots$, $W^{l}$, using kinds of subjective and objective assigning weights methods. For example, one of the weight vector is $W^{k}=\left(w_{1}{ }^{k}, w_{2}{ }^{k}, \mathrm{~L}, w_{m}{ }^{k}\right)^{T}$, it meets the equation $\sum_{j=1}^{m} w_{j}{ }^{k}=1, w_{j}{ }^{k} \geq 0, \quad(k=1,2, \mathrm{~L}, l)$.

Make the vector $W^{*}=\left(w_{1}{ }^{*}, w_{2}{ }^{*}, \mathrm{~L}, w_{m}{ }^{*}\right)^{T}$ as the linear combination weight vector of the above several weight vectors such as $W^{1}, \ldots, W^{k}$. The formula (7) is about the linear combination weight vector.

$$
W^{*}=\sum_{k=1}^{l} x_{k} W^{k}
$$

In the above formula, the variable $x_{k}$ stands for the linear combination coefficient, and it accords with the equation

$$
\sum_{k=1}^{l} x_{k}=1, \quad x_{k} \geq 0
$$

Order the vector $x=\left(x_{1}, x_{2}, \mathrm{~L}, x_{l}\right)^{T}$ stands for the linear combination coefficient vector, and it can be easily verified that the weight vector drawn from the formula (7) accords with the equation

$$
\sum_{j=1}^{m} w_{j}^{*}=\sum_{j=1}^{m} \sum_{k=1}^{l} x_{k} w_{j}^{k}=1
$$

(2) From the point of view of mathematical statistics, in the realistic system, the true weight coefficient of every evaluation index is a random variable, and the true weight vector that they form is a random vector. The weight vector $W^{k}$ can be viewed as a sample of the true weight vector, and the linear combination coefficient $x_{k}$ can be viewed as the probability of the true weight vector taking the sample value. So the linear combination coefficient vector $x$ has some uncertainty and this uncertainty can be shown by the Shannon entropy:

$$
H=-\sum_{k=1}^{l} x_{k} \ln x_{k}
$$

(3) The purpose of solving for the linear combination vector is just to confirm the suitable combination coefficient vector $x$. So on one hand it should make the weighting "bull's-eye distances" between all schemes and the ideal scheme minimum, which means the two follow formulas should be tenable.

$$
\begin{gathered}
\text { Min } \sum_{i=1}^{n} d_{i}^{2}=\sum_{i=1}^{n} \sum_{j=1}^{m} \sum_{k=1}^{l} x_{k} w_{j}^{k}\left(r_{j}^{*}-r_{i j}\right)^{2} \\
\text { s.t. } \quad \sum_{k=1}^{l} x_{k}=1, \quad x_{k} \geq 0
\end{gathered}
$$

And on the other hand, it should try its best to dispel the uncertainty with the combination coefficient vector $x$. According to Jayne's greatest entropy principle, the comprehensive weight coefficient for index should make the Shannon entropy unable to fetch great, it means that the two follow formulas should be tenable. 


$$
\begin{aligned}
& \operatorname{Max} \quad H=-\sum_{k=1}^{l} x_{k} \ln x_{k} \\
& \text { s.t. } \quad \sum_{k=1}^{l} x_{k}=1, \quad x_{k} \geq 0
\end{aligned}
$$

So solving for the linear combination weight vector is a problem of multiple attributes optimization. In order to solve this problem, this text constructs the follow single attribute optimization problem (SP):

$$
\begin{gathered}
\operatorname{Min} \sum_{i=1}^{n} \sum_{j=1}^{m} \sum_{k=1}^{l} x_{k} w_{j}^{k}\left(r_{j}^{*}-r_{i j}\right)^{2}+(1-\mu) \sum_{k=1}^{l} x_{k} \ln x_{k} \\
\text { s.t. } \quad \sum_{k=1}^{l} x_{k}=1, \quad x_{k} \geq 0
\end{gathered}
$$

In the formula (15), the positive parameter $0<\mu<1$ expresses the balance coefficient between two attributes, and it can be provided in advance according to the practical problem. It can be proved that this single attribute optimization problem (SP) has the only result and the result is as follows:

$$
x=\left[\frac{p_{1}}{p}, \frac{p_{2}}{p}, \mathrm{~L}, \frac{p_{l}}{p}\right]
$$

In the formula (17), the results of vector $p$ and vector $p_{k}$ are as follows:

$$
p=\sum_{k=1}^{l} p_{k}, \quad p_{k}=\exp \left\{-\left[1+\mu \sum_{i=1}^{n} \sum_{j=1}^{m} w_{j}{ }^{k}\left(r_{j}^{*}-r_{i j}\right)^{2} /(1-\mu)\right]\right\}, \quad(k=1,2, \mathrm{~L}, l)
$$

(4) Use the formula (7) to solve for the linear combination weight vector $W^{*}$.

\subsection{The multiple attribute grey target decision making algorithm}

In sum, it can get the multiple attribute grey target decision making algorithm as follows:

(1) Construct the effect sample matrix $X=\left[x_{i j}\right]_{n \times m}$ according to the multiple attribute decision making problem;

(2) Use formulas (1), (2), (3) to transform the effect sample matrix $X$ into the decision making matrix $R=\left[r_{i j}\right]_{n \times m}$;

(3) Solve for the optimum effect vector $r$ according to the formula (4) by the decision making matrix $R$;

(4) Solve for the linear combination coefficient vector $x$ according to the formula (17);

(5) Solve for the linear combination weight vector $W^{*}$ according to the formula (7);

(6) Solve for the "bull's-eye distance" $d_{i}$ of the effect vector $r_{i}$, and arrange $d_{i}$ according to the order from small to large, then can get the optimum sequencing of every scheme.

\section{Demonstrated application}

\subsection{Brief account of the application}

In order to develop the new products, five kinds of capital schemes from $s_{1}$ to $s_{5}$ have been drafted. The effect sample value of every scheme is arranged in Table 1, and tries to arrange them in an order.

\subsection{The model calculation}

Among the indexes, the Expect NPV and Risk Profit are benefit type indexes while the Capital Cost and Risk Loss are cost type indexes. We utilize the method of this text to solve for the sequencing of the capital schemes, and the concrete steps are as follows:

(1) Set up the effect sample matrix according to data of table 1;

$$
X=\left[\begin{array}{cccc}
5.20 & 5.20 & 4.73 & 0.473 \\
10.08 & 6.70 & 5.71 & 1.599 \\
5.25 & 4.20 & 3.82 & 0473 \\
9.72 & 5.25 & 5.54 & 1.313 \\
6.60 & 3.75 & 3.30 & 0.803
\end{array}\right]
$$


(2) Transform the effect sample matrix into the decision making matrix according to formula (1) and formula (2);

$$
R=\left[\begin{array}{cccc}
0.8007 & 0.1071 & 0.0833 & 0.6886 \\
-1 & 1 & 0.8257 & -1 \\
0.7823 & -0.4881 & -0.6060 & 0.6886 \\
-0.8671 & 0.1369 & 0.6969 & -0.5711 \\
0.2841 & -0.7559 & -1 & 0.1937
\end{array}\right]
$$

(3) Get the optimum effect vector according to the formula (4);

$$
r=(0.8007,1,0.8257,0.6886)
$$

(4) Supposed that the appraising person gets three kinds of weight vectors for the evaluation index using different kinds of subjective and objective assigning weights methods, they are as follows:

$$
\begin{gathered}
W^{1}=(0.10,0.30,0.15,0.45) \\
W^{2}=(0.20,0.30,0.30,0.40) \\
W^{3}=(0.45,0.15,0.10,0.30)
\end{gathered}
$$

Make the balance coefficient $\mu=0.8$ and calculate the combination coefficient vector according to formula (17);

$$
x=(0.6665,0.0039,0.3296)
$$

(5) Solve for the linear combination weight vector according to formula (7);

$$
W^{*}=(0.216,0.251,0.134,0.399)
$$

(6) Solve for the "bull's-eye distance" $d_{i}$ of every scheme;

$$
d_{1}=0.5234, d_{2}=1.3558, d_{3}=0.9114, d_{4}=1.1930, d_{5}=1.1730
$$

(7) Arrange $d_{i}$ according to the order from small to large, then can get the optimum sequencing of every scheme.

$$
d_{1}>d_{3}>d_{5}>d_{4}>d_{2}
$$

3.3 The result analysis

Table 2 shows the comparison of assessment results of different assigning weights methods. Seeing from table 2, the different methods all get the result of $s_{1}>s_{3}$, so it can be thought that scheme 1 is optimum and scheme 2 takes second place. The assessment results of the other three schemes are not the same, so it can't well offer reference for making policy though single one assigning weights method. The linear combination assigning weights method adopted by this text can synthesize characteristics of various kinds of methods so better as to offer basis for making policy.

\section{Conclusion}

The choice of the weight coefficient of the evaluation index is the critical event while appraising multiple attributes system. Using optimization theory and Jayne's maximum entropy principle a method of linear combination weights assigning based on entropy is presented to deal with the problem of assigning weights in multiple attributes decision making, it considers not only the synthesis of subjective assigning weights method and objective method but also the uncertainty of different methods, and the existing grey target decision making model has been got improved. At last the demonstrated application shows the rationality and significance of this method.

\section{References}

Chen, Huayou. (2003). Research on Optimal Combination Determining Weights Method for Multiple Attribute Decision Making. Operations Research and Management Science. vol. 012, no. 02, pp. 06-10.

Dai, Wenzhan, Zou, Lihua, Wang, Jianzhang, Zhu, Jingang \& Zhou, Rong. (2000). A Method of the Dynamic Multiple Attribute Decision Modeling Based on New Effective Function and Its Application. System Engineering Theory and Practice. vol. 020, no. 06, pp. 32-36.

Feng, Ao, Liu, Bin \& Zhu, Nan. (2000). Analysis and Application of Multi-Index System. MINI-MICRO SYSTEM. vol. 021 , no. 05 , pp.527-529.

Hou, Chang. (1995). Grey Decision on Appraisal of Original Buildings. Industrial Construction. vol. 025, no. 007, pp. 07-10.

Wang, Wenping. (1997). A Study of the Grey Utility Theory for Grey Target Decision. J, Huazhong Univ, of Sci, \& Tech, vol. 025(supplementary issue), pp. 89-91.

Xiang, Yuelin. (1996). A Grey Target Decision Method for Selecting Investment Direction of Pollution Control. 
Chemical Environmental Protection. vol. 016, no. 02, pp. 102-106.

$\mathrm{Xu}$, Zeshui \& Da, Qingli. (2002). Study on Method of Combination Weighting. Chinese Journal of Management Science. vol. 010, no. 02, pp. 84-87.

Table 1. Effect sample value of every sample Unit: ten thousands of RMB

\begin{tabular}{|l|l|l|l|l|}
\hline $\begin{array}{c}\text { Target } \\
\text { Scheme }\end{array}$ & Capital Cost & Expect NPV & Risk Profit & Risk Loss \\
\hline$S_{1}$ & 5.2 & 5.2 & 4.73 & 0.473 \\
\hline$S_{2}$ & 10.08 & 6.7 & 5.71 & 1.599 \\
\hline$S_{3}$ & 5.25 & 4.2 & 3.82 & 0.473 \\
\hline$S_{4}$ & 9.72 & 5.25 & 5.54 & 1.313 \\
\hline$S_{5}$ & 6.6 & 3.75 & 3.3 & 0.803 \\
\hline
\end{tabular}

Table 2. Assessment results of different assigning weights methods

\begin{tabular}{|c|c|c|c|c|}
\hline $\begin{array}{l}\text { Weight Vector } \\
\text { Scheme }\end{array}$ & $W^{1}$ & $W^{2}$ & $W^{3}$ & $W^{*}$ \\
\hline$s_{1}$ & 0.56732 & 0.63603 & 0.41798 & 0.283289 \\
\hline$s_{2}$ & 1.26782 & 1.33755 & 1.52136 & 1.151147 \\
\hline$s_{3}$ & 0.98581 & 1.13107 & 0.73300 & 0.501505 \\
\hline$S_{4}$ & 1.10372 & 1.191431 & 1.35689 & 1.156536 \\
\hline$S_{5}$ & 1.24973 & 1.44092 & 0.99467 & 0.936148 \\
\hline $\begin{array}{l}\text { Result of Optimum } \\
\text { Sequence }\end{array}$ & $S_{1}>S_{3}>S_{4}>S_{5}>S_{2}$ & $S_{1}>S_{3}>S_{4}>S_{2}>S_{5}$ & $S_{1}>S_{3}>S_{5}>S_{4}>S_{2}$ & $s_{1}>S_{3}>S_{5}>S_{2}>S_{4}$ \\
\hline
\end{tabular}

